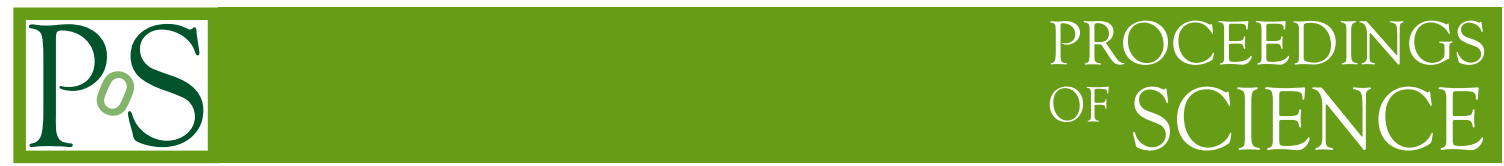

\title{
New measurements in the quarkonia sector
}

\section{Valeriia Zhovkovska ${ }^{a, *}$ on behalf of the ALICE, ATLAS, CMS and LHCb \\ Collaborations}

\author{
${ }^{a}$ Université Paris-Saclay, \\ CNRS/IN2P3, \\ IJCLab, \\ 15 rue Georges Clémenceau, Orsay, France \\ E-mail: valeriia.zhovkovska@cern.ch
}

An overview of recent measurements by the LHC experiments in the quarkonia sector is presented in this proceedings. Selected results on differential production cross-section, P-wave charmonia polarization and associated quarkonia production are discussed.

The Ninth Annual Conference on Large Hadron Collider Physics - LHCP2021

7-12 June 2021

Online

$$
{ }^{*} \text { Speaker }
$$




\section{Introduction}

Quarkonium is a bound state of a quark and its own antiquark, composed only of heavy quarks $q$ which mass $m_{q}$ is bigger than the binding energy. It plays an important role in the understanding of strong interaction. In $p p$ collisions, quarkonium state can be produced directly from hard collisions of partons (hadroproduction), through the feed-down of excited states, or via decays of $b$-hadrons. The quarkonia from the first two sources originate from the primary vertex of the $p p$ collision, and are called prompt, while those from the last source originate from $b$-decay vertices, which are typically displaced from the primary vertex, and are called charmonia from- $b$. At present, the effective quantum field theory of non-relativistic Quantum Chromodynamics (NRQCD) constitutes the most powerful approach to describe quarkonium production.

The simplest approach assumes that only heavy quark pairs produced in the dominant Fock state form a physical quarkonium. It considers that the colour of the $q \bar{q}$ can be the same as the physical final state $H$, and the colourless state is then called a color singlet state. Therefore, the model which describes this process is called Colour-Singlet model.

The NRQCD approach provides a natural solution by generalizing Colour-Singlet model (CS) to Colour-Octet (CO) model. In this model, heavy quark pairs that are produced at short distances in a colour-octet state can emit soft gluons at a later stage of the production process, when the quark pair has already expanded to the quarkonium size. According to the power counting rules, $\mathrm{CO}$ matrix elements for S-wave quarkonia are suppressed by power of the velocity compared to the CS contribution. Nevertheless, the $\mathrm{CO}$ processes can be dominant in case of enhanced production of $q \bar{q}$ in a $\mathrm{CO}$ state.

This proceeding focuses on the selected quarkonia production and polarization measurements in $p p$ collisions from the ALICE, ATLAS, CMS and LHCb experiments. The LHC experiments have complementary acceptance ranges. The ATLAS, CMS and ALICE experiments perform measurements in midrapidity range, at the same time ALICE and LHCb experiments cover forwardrapidity. All the experiments can access dimuon $\left(\mu^{+} \mu^{-}\right)$final state, when the LHCb experiment can also perform precise measurements via hadronic final states.

\section{Quarkonia production}

The most precise charmonium studies employ decays into experimentally clean dilepton final states, which is possible for $J^{P C}=1^{--}$charmonia, such as $J / \psi$ and $\psi(2 S)$ states. The ATLAS experiment reported a measurement of $J / \psi$ and $\psi(2 S)$ prompt production cross-section and production from $b$-decays at $\sqrt{s}=13 \mathrm{TeV}$ [1]. The charmonia yields are measured for rapidity $|y|<2.0$ and transverse momentum $60<p_{\mathrm{T}}<360 \mathrm{GeV} / c$ in the dimuon channel. $J / \psi$ inclusive production was also studied by the ALICE experiment at $\sqrt{s}=5.02 \mathrm{TeV}$ in $|y|<0.9$ (Fig. 1) [2]. At the same time the $\mathrm{LHCb}$ experiment reported $\psi(2 S)$ production measurement at $\sqrt{s}=7$ and $13 \mathrm{TeV}$ in a complementary kinematical region: $2.0<|y|<4.5$ and $p_{\mathrm{T}}<20 \mathrm{GeV} / c$ (Fig. 2) [3]. For the $J / \psi$ and $\psi(2 S)$ prompt production rates, the next-to-leading order (NLO) calculations in NRQCD can successfully describe the measurements in, however, a limited range of transverse momentum. The measured charmonium production in $b$-decays is is well-described by FONLL predictions [4].

The first measurement of the $\eta_{c}$ meson hadroproduction at $\sqrt{s}=7 \mathrm{TeV}$ [5] revealed a lack of comprehensive theoretical model able to simultaneously describe a production cross-section of the 


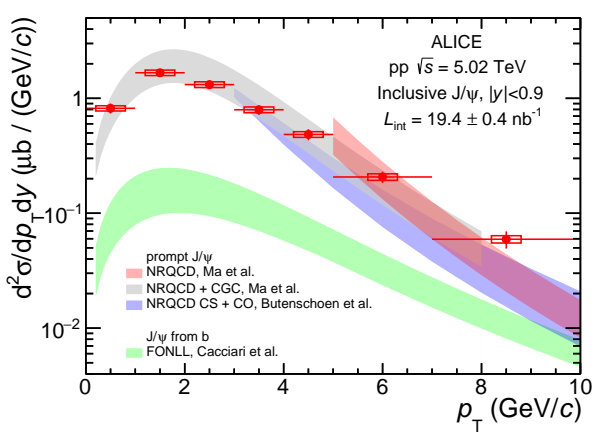

Figure 1: Inclusive $J / \psi \quad p_{\mathrm{T}}$-differential crosssections [2].

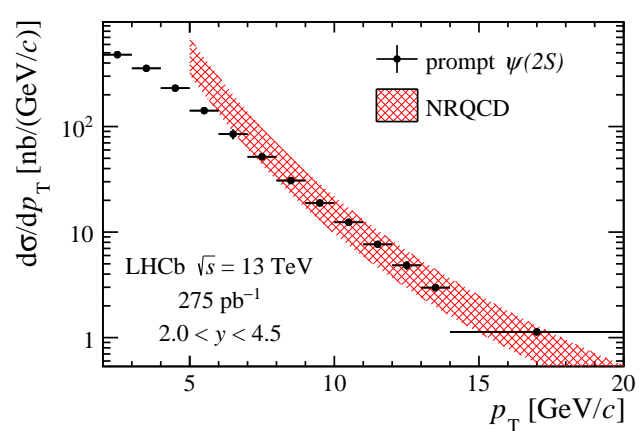

Figure 2: Prompt $\psi(2 S) p_{\mathrm{T}}$-differential crosssections at $13 \mathrm{TeV}$ [3].

$J / \psi$ and $\eta_{c}(1 S)$ states and a polarisation of the $J / \psi$ meson. The $\eta_{c}(1 S)$ production measurement by the LHCb Collaboration triggered a major revisiting of the theoretical framework and yielded new approaches capable to describe simultaneously the three observables, however, in a limited $p_{T}$ range. The LHCb experiment published a measurement of the $\eta_{c}(1 S)$ production at $\sqrt{s}=13 \mathrm{TeV}$ for $6.5<p_{\mathrm{T}}<14 \mathrm{GeV} / c$ and $2.0<|y|<4.5$ [6]. It shows that $\eta_{c}(1 S)$ prompt production can be described by CS contribution (Fig. 3 (b)). New studies in the extended $p_{\mathrm{T}}$ region will provide an improved sensitivity to a possible $\mathrm{CO}$ contribution.

\section{Quarkonia polarization}

Another important observable is charmonia polarization. The CMS Collaboration reported the $\chi_{c 2} / \chi_{c 1}$ polarization ratio as a function of $\left|\cos \theta^{H X}\right|$ and $\phi^{H X}$ [7]. The $\chi_{c}$ states are reconstructed via their radiative decays $\chi_{c} \rightarrow J / \psi \gamma$, with the photons being measured through conversions, which allows the two states to be well resolved. While azimuthal decay angle distributions show no differences between the two states, polar anisotropies are observed to be significantly different. A scenario where both states are unpolarized along the helicity quantization axis was excluded at a $99.7 \%$ confidence level. This is the first measurement of significantly polarized quarkonia produced at high transverse momentum and it shows good agreement with NRQCD predictions (Fig. 4).

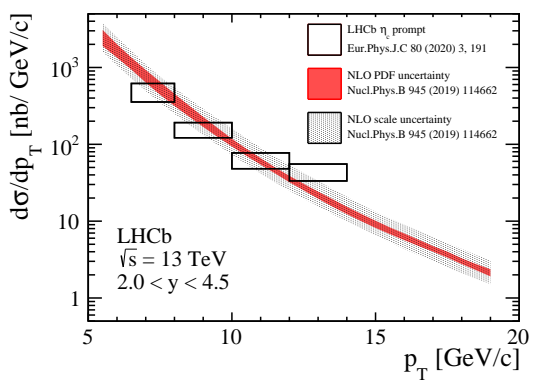

Figure 3: Relative $\eta_{c} / J / \psi$ prompt production cross-section (boxes), NLO NRQCD prediction with PDF (red band) and scale (grey band) uncertainties.

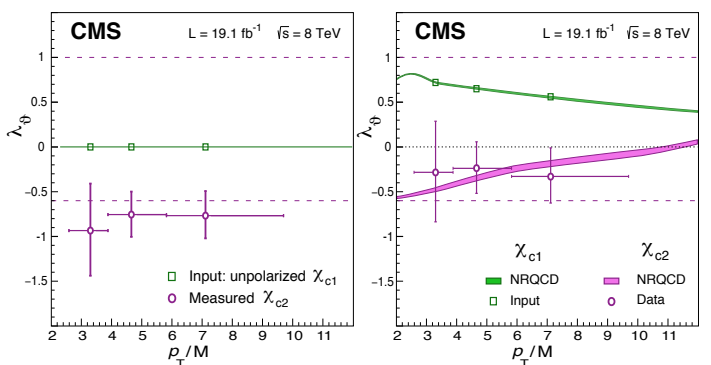

Figure 4: The $\chi_{c 2}$ polarization values (circles) measured when the $\chi_{c 1}$ polarization values (squares) are fixed to the unpolarized (left) or the NRQCD (right) scenarios (green curves), as a function of $p_{\mathrm{T}} / \mathrm{M}$ of the $J / \psi$ [7]. 


\section{Associated production}

In order to test the charmonium hadronization mechanism, the CMS experiment studied $J / \psi$ production inside jets at $\sqrt{s}=8 \mathrm{TeV}$ [8]. The analysis combines the experimental measurement of $J / \psi$ mesons contained in jets with a theoretical approach based on the fragmenting jet function (FJF) model [9]. The FJF model postulates that the $c \bar{c}$ pair is produced in fragmentation of a high- $p_{\mathrm{T}}$ jet. The cross-section contributions for all relevant ${ }^{2 S+1} L_{J}^{n}$ terms are computed using the NRQCD methodology. Each cross-section term has a characteristic relation between the jet energy $E_{j e t}$ and its fraction carried by the $J / \psi$ meson: $z=E_{J / \psi} / E_{j e t}$. The differential cross-section as a function of $E_{j e t}$ is measured in three ranges of $z$ and compared to the FJF prediction for this quantity, using three different long-distance matrix elements (LDME) parameter sets. The FJF prediction using the BCKL [10] LDME parameters shows the best agreement with the data for all three $z$ ranges.

The ATLAS experiment measured the ratio of the associated prompt $J / \psi$ and $W^{ \pm}$production cross-section to the inclusive $W^{ \pm}$boson production cross-section at $\sqrt{s}=8 \mathrm{TeV}$ [11]. The crosssection ratios are reported for $8.5<p_{T}^{J / \psi}<150 \mathrm{GeV} / c$ and $|y|<2.1$. Study of the opening angle $\Delta \phi\left(J / \psi, W^{ \pm}\right)$for prompt $J / \psi+W^{ \pm}$candidates suggest presence of both single- and double-partonscattering contributions (SPS and DPS). The result is compared with a theoretical prediction at NLO for CO prompt production processes. Two different values of the effective DPS cross-section $\sigma_{e f f}$ are used. However, neither of them can correctly describe $J / \psi p_{\mathrm{T}}$-dependence.

The CMS experiment reported another important probe of QCD processes using quarkonium pair production. The $\Upsilon(1 S)$ pair production was measured at $\sqrt{s}=13 \mathrm{TeV}$ with four muons in the final state and where both $\Upsilon(1 S)$ have an absolute rapidity $|y|<2.0$ [12]. The DPS contribution to the total inclusive pair production cross-section is estimated for the first time to be $(39 \pm 14) \%$. This analysis also aims at searching for narrow resonances decaying to $\Upsilon(1 S) \mu^{+} \mu^{-}$. No excess of events compatible with a signal is observed in the four-muon invariant mass spectrum. Upper limits at $95 \%$ confidence level on the product of the signal cross-section and branching fraction to four muons via an intermediate resonance are set for different signal models, expanding the kinematic and mass coverage of previous searches [13].

A similar study of $J / \psi$ pair production was performed by the LHCb experiment at $\sqrt{s}=7$ and $8 \mathrm{TeV}$ in order to search for a possible tetraquark candidate [14]. A narrow structure, $X(6900)$, matching the lineshape of a resonance and a broad structure next to the di-J/ $\psi$ mass threshold are found. The global significance of both structures is estimated to be larger than five standard deviations. The narrow $X(6900)$ structure is a possible four-charm tetraquark candidate. The broad structure close to the di- $J / \psi$ mass threshold could originate from a mixture of multiple four-charm quark states or have contributions from feed-down decays of four-charm states through heavier quarkonia. Additional study with more data and spin-parity determination are required to understand the nature of the observed structure.

\section{Summary}

The summary of recent measurements in the quarkonia sector performed at LHC has been presented. It covers studies of different observables important for understanding the quarkonia production mechanism. The LHC experiments provide measurements in complementary kinematical ranges and different final states. Improved statistics and new datasets would allow to improve the precision of the existing measurements and access new observables for the quarkonia studies. 


\section{References}

[1] ATLAS collaboration, Measurement of the production cross-section of $J / \psi$ and $\psi(2 S)$ mesons at high transverse momentum in pp collisions at $\sqrt{s}=13 \mathrm{TeV}$ with the ATLAS detector, ATLAS-CONF-2019-047 .

[2] ALICE collaboration, Inclusive J/ $\psi$ production at mid-rapidity in pp collisions at $\sqrt{s}=5.02 \mathrm{TeV}$, JHEP 10 (2019) 084 [1905.07211].

[3] LHCв collaboration, Measurement of $\psi(2 S)$ production cross-sections in proton-proton collisions at $\sqrt{s}=7$ and 13 TeV, Eur. Phys. J. C80 (2020) 185 [1908.03099].

[4] M. Cacciari, S. Frixione, N. Houdeau, M.L. Mangano, P. Nason and G. Ridolfi, Theoretical predictions for charm and bottom production at the LHC, JHEP 10 (2012) 137 [1205. 6344].

[5] LHCв collaboration, Measurement of the $\eta_{c}(1 S)$ production cross-section in proton-proton collisions via the decay $\eta_{c}(1 S) \rightarrow p \bar{p}$, Eur. Phys. J. C75 (2015) 311 [1409.3612].

[6] LHCв collaboration, Measurement of the $\eta_{c}(1 S)$ production cross-section in pp collisions at $\sqrt{s}=13$ TeV, Eur. Phys. J. C80 (2020) 191 [1911.03326].

[7] CMS collaboration, Constraints on the $\chi_{\mathrm{c} 1}$ versus $\chi_{\mathrm{c} 2}$ Polarizations in Proton-Proton Collisions at $\sqrt{s}=8 \mathrm{TeV}$, Phys. Rev. Lett. 124 (2020) 162002 [1912 . 07706].

[8] CMS collaboration, Study of J/4 meson production inside jets in pp collisions at $\sqrt{s}=8 \mathrm{TeV}$, Phys. Lett. B804 (2020) 135409 [1910 . 01686].

[9] M. Baumgart, A.K. Leibovich, T. Mehen and I.Z. Rothstein, Probing Quarkonium Production Mechanisms with Jet Substructure, JHEP 11 (2014) 003 [1406.2295].

[10] G.T. Bodwin, H.S. Chung, U.-R. Kim and J. Lee, Fragmentation contributions to J/ $\psi$ production at the Tevatron and the LHC, Phys. Rev. Lett. 113 (2014) 022001 [1403 . 3612].

[11] ATLAS collaboration, Measurement of $J / \psi$ production in association with a $W$ boson with pp data at $8 \mathrm{TeV}$, JHEP 01 (2020) 095 [1909. 13626].

[12] CMS collaboration, Measurement of the $\Upsilon(1 S)$ pair production cross section and search for resonances decaying to $\Upsilon(1 S) \mu^{+} \mu^{-}$in proton-proton collisions at $\sqrt{s}=13 \mathrm{TeV}$, Phys. Lett. B808 (2020) 135578 [2002.06393].

[13] CMS collaboration, Observation of $\Upsilon(1 S)$ pair production in proton-proton collisions at $\sqrt{s}=8 \mathrm{TeV}$, JHEP 05 (2017) 013 [1610.07095].

[14] LHCв collaboration, Observation of structure in the J/ $\psi$-pair mass spectrum, Sci. Bull. 65 (2020) 1983 [2006 . 16957]. 